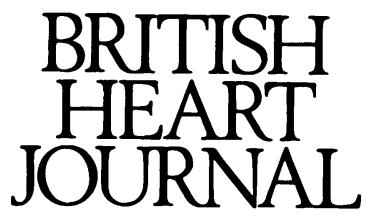

\title{
COMMENTARY
}

\section{Potential for antiviral therapy in the treatment of restenosis after angioplasty}

Two papers in Science in 1994 are landmark studies in the investigation of restenosis after angioplasty. Both raise the possibility that antiviral therapy may be a treatment for this important clinical problem.

The protein p53 is a regulator of cell growth and usually has a short half life. Mutation of the p53 gene resulting in loss of its tumour suppressive activity is seen in up to $50 \%$ of human cancers. The mutation of the gene also enhances the stability of the protein allowing it to be identified immunohistochemically in tumour cells and this property is being proposed as a clinically useful prognostic indicator. Because the coronary restenotic lesion has features of a benign tumour with the exuberant proliferation of vascular smooth muscle cells Spier, working in Stephen Epstein's laboratory at the National Institute of Health, decided to look for p53 immunoreactivity in a series of atherectomy specimens from restenotic lesions. ${ }^{1}$ None of 20 primary coronary lesions contained p53 immunoreactivity, whereas $38 \%$ of 60 restenotic lesions contained $>10 \%$ of cells that stained positively. However, unlike cancer cells in which the p53 is of a mutant non-growth suppressive type, the p53 gene (identified by the polymerase chain reaction (PCR)) in the neointimal vascular smooth muscle cells was normal, raising the question why the protein is sufficiently stable to accumulate and be identified histochemically.

In some animal models Herpesvirus infection results in atherosclerotic proliferation of vascular smooth muscle cells. In the Spier study a PCR analysis of the human restenotic lesions revealed human Cytomegalovirus (HCMV) gene products in 85\% (11 of 13 examined) of those in which p53 had already been identified and only in 3 of 11 with p53 immunonegative lesions. Further studies in vitro of vascular smooth muscle cells infected with HCMV showed that p53 accumulated with a similar profile to one of the viral proteins, IE84, and cotransfection of the protein and p53 showed that the IE84 regulated p53 expression. Further studies suggested this is a protein-protein interaction. Thus the hypothesis is that injury-induced reactivation of latent HCMV infection, though not inducing true tumour formation, results in IE84 expression which inhibits degradation and the repressor function of $\mathrm{p} 53$ in the vascular smooth muscle cells thereby promoting the proliferation of vascular smooth muscle cells that is a feature of post-angioplasty restenosis.

Though this is an attractive hypothesis certain aspects require further clarification. Atherectomy specimens are extremely variable in their components, ranging from thrombus and cellular debris to highly cellular areas which may even be derived from the adventitia. Similarly, there are great differences in the nature of the tissue that can be derived from primary rather than restenotic lesions-primary lesions often yield little more than amorphous debri with few cells. It is not clear what contribution the different cellular elements in samples of restenotic (compared with primary) lesions make to the different detection rates of HCMV and p53. Also, neointimal proliferation occurs to some degree after all angioplasty or stenting procedures, but the development of angiographic or clinically relevant restenosis seems to be regulated by slow recoil of the stretched vessel and the degree of injury induced. It would be fascinating to examine vessels that have undergone angioplasty but not become "restenotic" to see whether they show similar HCMV and p53 profiles.

Several animal models of angioplasty consistently produce the hyperplastic intimal lesion without any evidence of a viral infection. Spiers and colleagues report that there was no expression of $\mathrm{p} 53$ in the neointimal vascular smooth muscle cells of the experimentally induced neointima in the rat; so clearly this is not an essential feature of injury caused by balloon catheters. This raises the question whether the observation is an epiphenomenon rather than a factor in pathogenesis. The attraction of the HCMV/IE84/p53 theory is that such a response would be open to manipulation by antiviral therapy.

Another group, that of Gary and Elizabeth Nabel, has also raised the possibility of applying antiviral therapy to the restenotic disease process. They intervened directly to induce susceptibility to the antiviral drug gancyclovir. ${ }^{2}$ Using a replication deficient adenovirus they transfected the injured vessels with the viral enzyme thymidine kinase. This enzyme converts the gancyclovir to a cytotoxic metabolite that kills only proliferating cells. An adenoviral vector has the advantage of having a high transfection efficiency but transient (weeks) expression of the gene. When gancyclovir was given, only the transfected cells that were actively proliferating were killed. The result, shown in the pig iliac model of injury induced by balloon catheter, was that neointimal formation was substantially inhibited. The major impetus for this roundabout approach is that adenovirus vectors are already being examined in other gene therapy programs, notably in that targeting cystic fibrosis. Adenovirus vaccination has been used successfully and safely by the US military and clinical protocols are already being examined using related systems in brain tumours, so approval for clinical trials in angioplasty restenosis is likely to be forthcoming from the Food and Drug Administration.

Hatter Institute for Cardiovascular Studies,

JEAN MCEWAN

University College London Medical School,

London WD1E 6 Ff

1 Spier E, Modali R, Huang E-S, Leon MB, Shawl F, Finkel T, Epstein SE. Potential role of human cytomegalovirus and $\mathrm{p} 53$ interaction in SE. Potential role of human cytomegalovirus and

2 human coronary restenosis. Science 1994;255:391-4. EG. Gene therapy for vascular smooth muscle cell proliferation after arterial injury. Science 1994;265:781-4.
a 